\title{
The Advancement of Virtual Reality in Automotive Market Research: Challenges and Opportunities
}

Alexandre Costa Henriques ${ }^{\mathrm{a}}$; Ingrid Winkler ${ }^{\mathrm{a}^{*}}$

a) SENAI CIMATEC, Salvador, Brazil. Email: alexandre.henriques@aln.senaicimatec.edu.br; ingrid.winkler@doc.senaicimatec.edu.br

* Corresponding author

\begin{abstract}
Virtual reality (VR) can play a key role in automotive marketing research, lowering costs and shortening the time it takes to bring a new product to market. However, there are still few VR applications that support automotive customers' experiences during the early stages of product development. Through a systematic review of literature and patents, this study aims to identify the challenges and opportunities for the application of virtual reality in car clinics, and to categorize them into attributes. We searched through the knowledge databases of PatentScout, ScienceDirect, Springer, and IEEEXplore. We found 72 patents with a high concentration in a few inventors. The United States of America presented the greatest number of records and the most common applications related to the apparatus for automatically reading respondents' reactions in a virtual environment. In terms of articles, we found 19 research papers that discussed sixteen categories identified as challenges and opportunities for automotive marketing research: 1) cost, 2) location to customers, 3) flexibility in interactions, 4) model transportation, 5) depth perception, 6) haptic perception, 7) motion, 8) movement perception/ physical collision, 9) color and texture, 10) sound feedback, 11) product interaction/manipulation, 12) visual-spatial, 13) graphic quality, 14) intuitiveness, 15) cybersecurity and 16) cybersickness. We conclude that the automotive industry can employ virtual reality for marketing research, but relevant elements such as hardware and software definition, stimulus quality, and research objectives, among others, must be considered.
\end{abstract}

Keywords: Virtual Reality; Automotive Industry; Marketing Research; Immersive Car Clinic.

\section{INTRODUCTION}

Virtual reality (VR) has been one of the most trendy technologies in the Industry 4.0 scenario. VR is defined as "a computer-generated digital environment that can be experienced and interacted with as if it were real" [1].

Because of the decreasing cost of hardware and software, as well as the wide range of applications, virtual reality has profit value, and companies are investigating innovative approaches. Thus, immersive technology usage is increasing in a variety of fields, including education [2], healthcare [3] [4], and construction [5]. 
Regarding the automobile market, the growth of VR applications is expected to be exponential in the coming years [6]. Automakers are using VR technology in manufacturing assessment [7], product development, dealer training, ergonomics evaluation, and customer engagement, among other applications.

Automakers conduct experiments with end consumers to learn about their opinions, preferences, and propensity for upcoming purchasing. These surveys, known as car clinics, deliver detailed customer feedback and insights to Marketing, Engineering, and Design departments [8].

Car clinics take place in a salon, where automakers display four to eight competing brands and models to customers. Assessments can be quantitative, in which enormous amounts of data are collected via questionnaires, or qualitative, in which customers are encouraged to express impressions based on close observation. The qualitative method is carried out with a small focus group, with a mediator guiding participants in obtaining answers on the study goal. Respondents may experience the vehicle's external and interior design, as well as compare content, at static automobile clinics. Participants are chosen based on a profile, which is often related to purchasing intentions.

Surveys are generally conducted using static full-scale physical models of the end product being studied to provide respondents with a closer approach to the final product. Vehicle size and appearance are key factors influencing customer product demands and purchasing decisions; thus, customer perception of in-scale items is critical for study in the automobile industry.

Prototypes are utilized for cars in the development phase, whereas production vehicles might be used for comparison. Such prototypes are built using specific manufacturing methods and need a large amount of labor from a skilled team. This comes at a high cost and requires careful planning during the automotive product development process.

Despite the numerous contemporary Virtual Reality applications in the automotive sector, there are still few VR applications that support the experiences of end automobile customers during the early product development phase. [9] identified various VR applications in the automobile industry, but they only investigated employees who are current or likely automaker users, not end automotive customers. [10] observed that the automotive industry exposes its products to customers in a virtual environment, but not during the product production phase. In the pre-purchase phase, automotive apps use Virtual Reality or Augmented Reality, such as advertising personalization based on face expression, car visualization, virtual racing tracking experience, and virtual tour of the car. The authors also noted that automotive companies may employ virtual technologies to improve consumer experiences in-store or on-premises. Despite research indicating the value of virtual reality with customers in the automotive industry, virtual reality with consumers has been restricted in the product development phase. 
Customers are more demanding and thirstier for new products, and market volume potential, fragmented automotive marketplaces, and other reasons have raised pressure on automakers to improve time to market with more accurate product definition. Virtual reality is important in marketing research since it reduces expenses and improves product development timing. Furthermore, VR applications may avoid costly stimulus rework and the need for specialist resources on standby mode during automobile clinics, which are frequently necessary in the event of property damage.

To date, no investigation of scientific and technological knowledge bases has been undertaken on employing virtual reality for automotive market research. [10] reviewed the literature on this subject, but they only examined scientific research articles and did not look at patent filings. These prospective studies are relevant for scientific and technical mapping since the utilization of patent sources of information combines the scope of technology management and innovation activities, constituting competitive tools used in the appraisal of technological potential [11] [12]. Furthermore, patents can contribute to the identification of people active in the field and support the decision-making of public and private institutions. The mapping made through scientific literature reveals the same possibilities. Furthermore, by analyzing scientific publications, it is possible to examine the state of the art in this technology, in addition to projecting probable trends and participating in the identification of new development research [13].

Therefore, this study aims to identify the challenges and opportunities and to categorize them into attributes for the application of virtual reality in car clinics through a systematic review of literature and patents.

The following is how this document is structured. Section 2 describes materials and methods adopted. Section 3 details and examines the findings. Finally, Section 4 provides our conclusions and recommendations for further research.

\section{MATERIALS AND METHODS}

This study is exploratory research, aiming to collect technical and qualified information with a patent and scientific article focus. The method is based on [14], divided into five stages: Planning, Scope, Search, Evaluation, and Synthesis.

\subsection{Planning}

First, we defined the following knowledge bases to search:

- Patent database: PatentScout

- Scientific articles databases: ScienceDirect, Springer, and IEEEXplore 
Databases must be evaluated according to some essential criteria for a search focusing on patents. Paid access tools, such as PatentScout, present as differentiators their speed in retrieving the information sought, their ability to analyze a large amount of data in a simplified and efficient way, the use of statistical tools, and promoting the generation of information through graphics and maps, among others, allowing analysis. The efficiency of this database can be directly related to the high cost of obtaining their license [15]. PatentScout has a wide-ranging database with several search options (general or advanced search) in different search fields. The recovery of documents can be made, besides keywords and Boolean operators, from the International Patent Classification (IPC) and the classifications elaborated by the database itself.

ScienceDirect, Springer, and IEEEXplore were chosen because they are reliable and multidisciplinary scientific databases of international scope with comprehensive coverage of citation indexing, allowing the best data from scientific publications.

\subsection{Scope}

In this study, two research questions were considered:

Q1: How has virtual reality (VR) supporting market research in the automotive industry?

Q2: What are the most recent advancements, challenges, and opportunities in the use of virtual reality for automotive market research?

\subsection{Searching}

We used the following keywords to search the PatentScout database: ("virtual reality" OR "virtual environment" OR "artificial environment") AND (Automotive OR Auto OR Car OR "Virtual Reality") AND ("Marketing Research" OR "Market Research" OR "Human Research" OR "People Research").

A similar search strategy was used for article retrieval, with minor adjustments to fit the search engine requirements of each scientific database: ("virtual reality" OR "virtual environment" OR "artificial environment") AND ("marketing research" OR "market research" OR "people research" OR "human research") AND document title with (automotive OR auto OR car OR "virtual reality"). Other criteria was used to restrict the results to only review or research articles and conference abstracts.

The search was carried out in July 2020, and the initial screening results revealed 72 patents and 1,089 publications. 


\subsection{Evaluation}

The evaluation stage aims to select the most relevant documents found. To narrow down the number of results, we assessed the documents facing the research questions and the application of automotive market research or virtual market research.

Authors' experience and knowledge in the investigated fields identified relevant documents connected with the two research questions. The Title and Abstracts of the articles as long the patent's summary was read, and documents were selected based on their relevance to:
a) Automotive sector application.
b) Human behavior in a virtual environment.
c) Opportunity of use in market research.
d) Virtual reality research with end users.

\subsection{Synthesis}

Ultimately, we selected for further analysis 72 patents and 13 scientific articles. These documents were exported to Microsoft Excel, where spreadsheets and graphics were subsequently created to analyze them.

\section{RESULTS AND DISCUSSION}

The patents were analyzed through their registration data source, inventors, subjective trends, technological advances, challenges, and opportunities. Articles were analyzed with a focus on identifying challenges, opportunities, and advances that can be applied to VR in automotive market research. Results of the patents and articles are exposed, and the two research questions are answered. A discussion on all the challenges and opportunities is performed.

\subsection{Patent Mapping}

The search identified 72 patent documents, of which only 37 are active. We analyzed patent publication number, title, abstract, active, assignee, inventors, priority date, publication date, file date, publication source, and first claim.

Figure 1 illustrates the trend in patent publications from 1995 to 2020. The first registration patent granted by the World Intellectual Property Organization (WIPO) has the subject of a computer system allowing a consumer to purchase packaged goods at home. From 2019 to 2020, results show a slight decrease in the publications concerning the year before. It is worth 
mentioning that publications may have been left out of the results because of the 18 months patent confidentiality period.

We found patents active from 2009 until 2020, reaching the highest value in 2018, with nine documents.

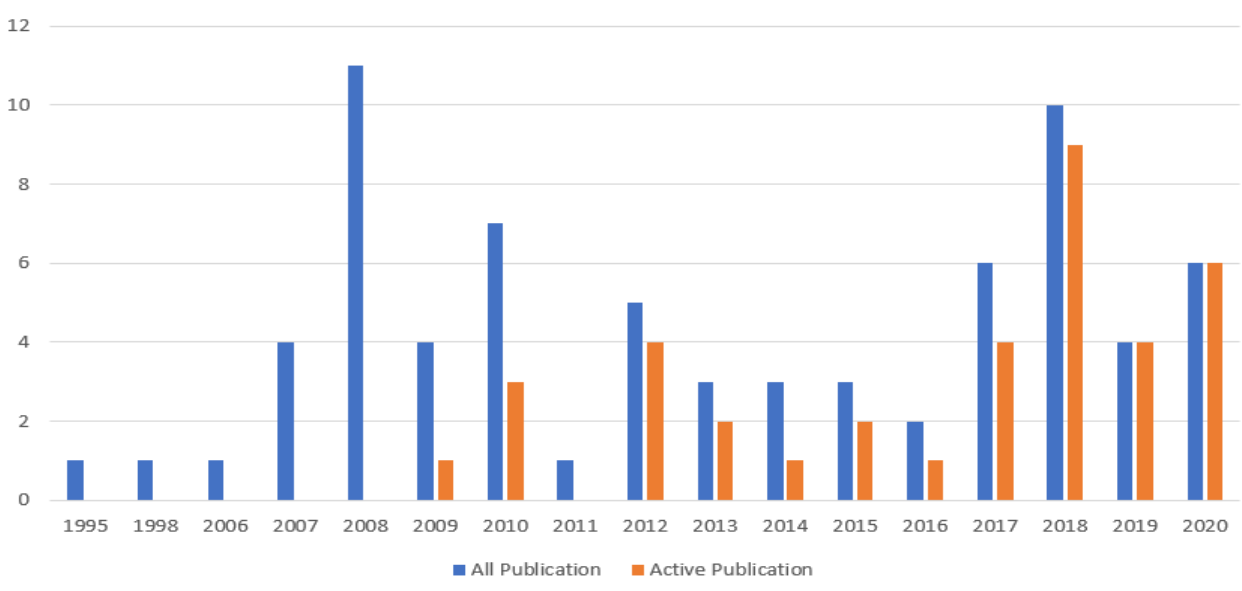

Fig. 1. Patent publishing trends between 1995 to 2020.

Regarding the publication source that contributes the most to the number of publications in prospective patent documents, the distribution of active patents worldwide is highly concentrated (Figure 2). The results showed that only four countries are responsible for the most significant interest and domain in the technology investigated in this study: The United States of America, Japan, India, and Chinese Taipei. Additionally, the United States has a significantly higher number of patents active than the other countries. In the segment of patents not active, we can also identify publication sources in the WIPO and European Patent Office, organizations representing multiple countries, as long as the Netherlands and South Korea.

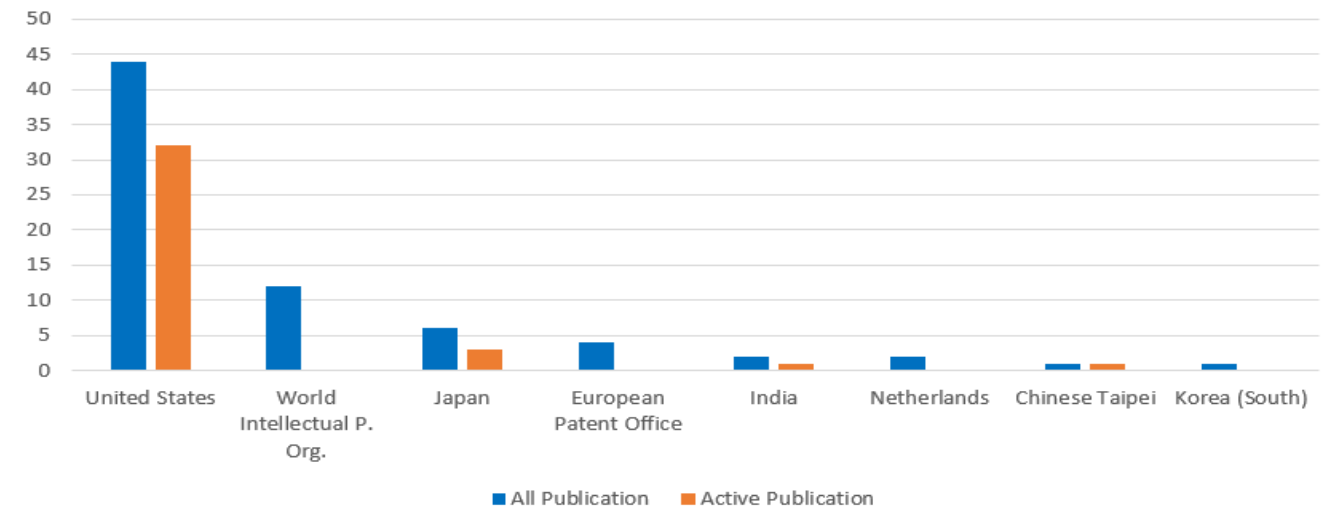

Fig. 2. Patent's origin source in terms of the number of publications. 
Regarding the patent inventor group, we identified 33 different inventor groups for the 72 patents. We found a high concentration of patents with a small group of inventors since only five different inventors' groups filled $46 \%$ of patents. 15 and 14 groups have four publications and each one represents six per cent of the total publications, 13 has six publications representing eight per cent, 12 has eight publications with $11 \%$ of the total while I1, with the most quantity of publications and a single inventor, achieve the value of 11 documents and represents $15 \%$ of the total publications. The other $54 \%$ patents can be grouped in inventors' groups with fewer than three publications each (Fig. 3).

Concerning active patents, five different inventors' groups correspond to $49 \%$ of the patents, with the United States owning four groups. The remaining $51 \%$ of the documents are applicable by different inventor's groups with a single application by a group.

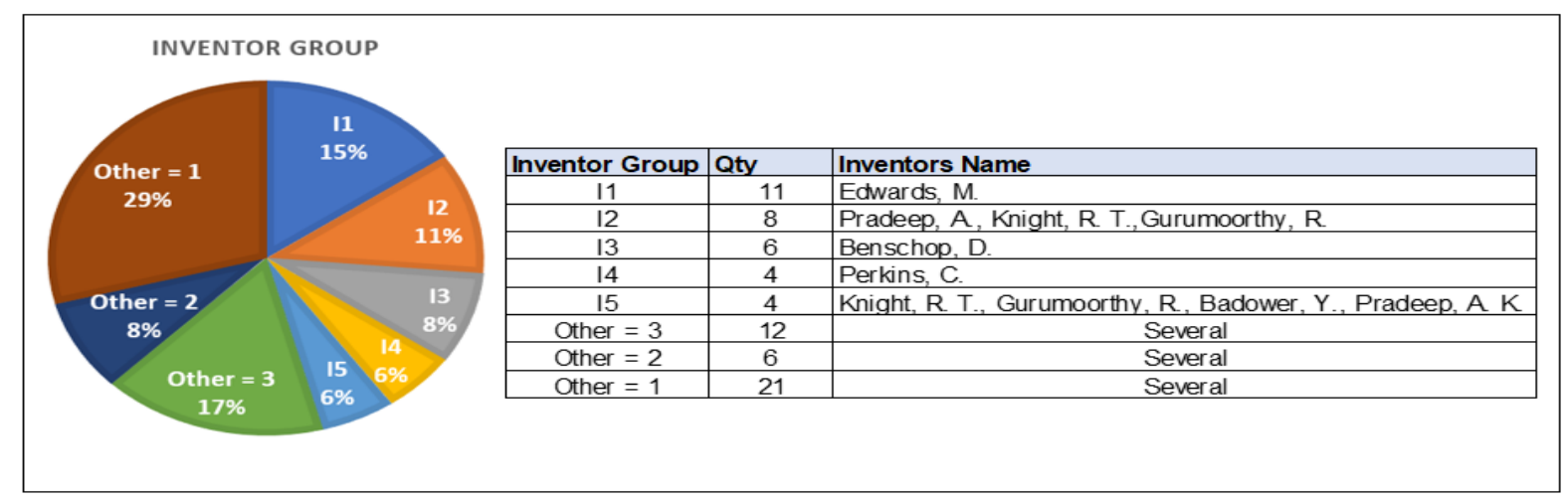

Fig. 3. Inventors group that most contribute in terms of the number of publications.

The patents could be grouped in the application, and authors divided them into four different categories: Virtual store, Customer profile, Advertisement, and Automatic Feedback. Virtual stores are patents related to the application of virtual reality in stores, Customer profiles are patents using virtual reality to identify the consumer profile, their intents of purchasing, etc. Advertisements are patents that use VR to advertise products/processes to the public and Automatic Feedback are patents related to the automatic interpretation of the user of VR through their changes in body positions, pupil changes, facial reactions, brainwaves, etc. In terms of classification by application, from 2009 to 2020, we identified patents related to virtual store applications in five patents from the United States and one from Chinese Taipei. Chinese Taipei, India, and Japan have one classification in customer profile while the United States has ten documents. Concerning advertisement, only Japan and the United States are represented in the Confidentiality Period with this classification with two and four applications, respectively. Automatic feedback in VR classification has only the United States of America represented by patents with 17 items. 
The analysis of the title and abstract of the active documents revealed that 17 documents correlate with apparatus to automatically read participants' reactions in a VR environment, such as their gestures, sounds, eye movements, or neurological signs. As Figure 4 illustrates, to automatically identify and analyze participants ' reactions is one subject that has been growing among the years on the patent side. As an example, US Patent $9,886,981$ defines a device that determines neurofeedback significance corresponding to stimulus material and then modifies the stimulus material using neuro-feedback significance measures [16].

Understanding customer profile applies to 13 documents such as US patent 9,881,310 that creates an eliciting system that may embody a method for collecting market research data by eliciting customer input through an interactive competitive game format [17].

Six patent applications related to VR are classified as advertising. For example, US patent $8,335,716$ with characteristics associated with multimedia advertisements are obtained in real-time and advertisement slots are offered with information on advertisement slot characteristics. They may be selected, purchased, exchanged, and analyzed by advertisers' corporations and firms [16].

Six patents are related to applications in virtual stores, such as the one presented in US patent $8,341,022$ where the invention is a virtual reality system that includes an instrumented device used to present a virtual shopping environment to a simulation participant [18].

Over the years, the most spread and stable application is the application on understanding customer profile, with publications from 2009 to recent years of the study. The advertisement application and virtual store are also stable and spread over the years but less frequently than customer profiles. Application on automatic feedback shows the biggest average and a growing profile.

It is important to remember that the counting of the last two years may be affected due to the 18-month patent confidentiality period (Fig. 4).

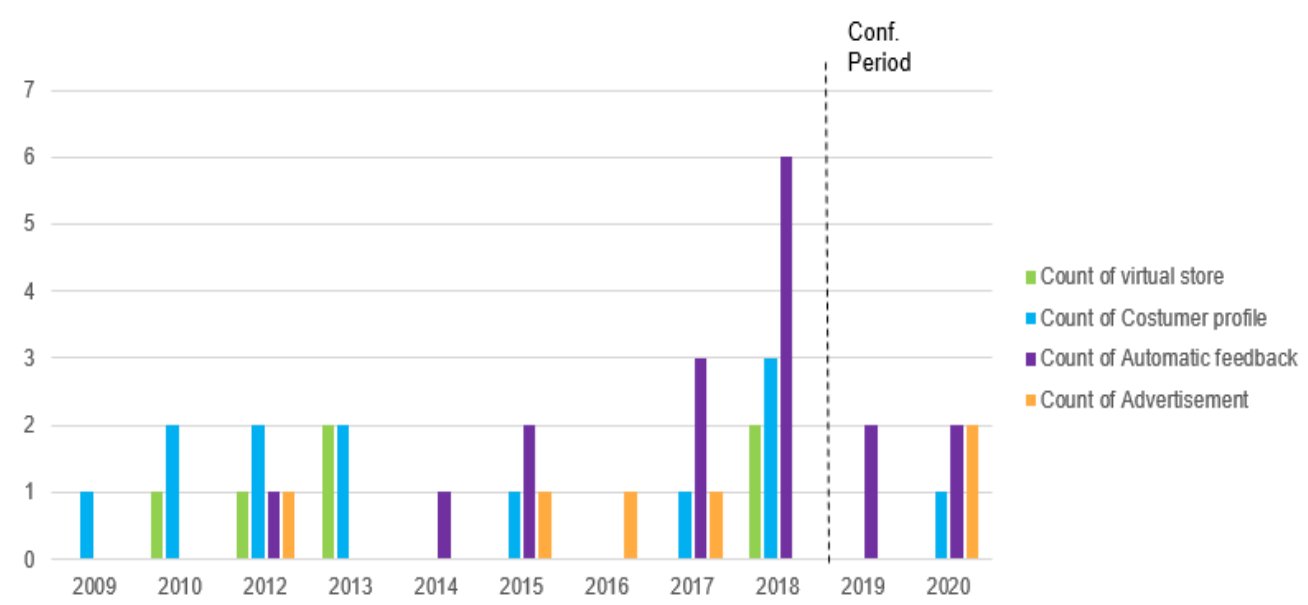

Fig. 4. Group classification over the years. 
The patent study provided clarity that virtual reality used to automatically identify and analyze participants ' reactions, such as eye pupil variation or neuro responses, is a recent advance of virtual reality with opportunities for automotive marketing research application as raised in Q2 (What are the most recent advancements, challenges, and opportunities in the use of virtual reality for automotive market research?) from the previous section.

\subsection{Scientific Mapping}

Search in the Web of Science, ScienceDirect, and IEEExplore knowledge databases identified 13 articles that may provide identification of challenges, opportunities, and advances that can be applied to VR in automotive market research. Table 1 shows these selected articles.

Table 1. List of Selected Studies.

\section{List of Selected Studies}

\begin{tabular}{lll}
\hline Reference & \multicolumn{1}{c}{ Title } & $\begin{array}{c}\text { Publication } \\
\text { Year }\end{array}$ \\
\hline$[9]$ & $\begin{array}{l}\text { Future directions for the development of virtual reality within an automotive } \\
\text { manufacturer }\end{array}$ & 2016 \\
\hline$[21]$ & Interaction techniques for virtual reality based automotive design reviews & 2017 \\
\hline$[22]$ & $\begin{array}{l}\text { Early prototype assessment of a new virtual system for training procedural skills of } \\
\text { automotive service operators: LARTE tool }\end{array}$ & 2015 \\
\hline$[20]$ & VR Processes in the Automotive Industry & 2015 \\
\hline$[27]$ & $\begin{array}{l}\text { Opportunities and constraints of virtual reality application in international and } \\
\text { domestic car companies of Malaysia }\end{array}$ & 2012 \\
\hline$[19]$ & $\begin{array}{l}\text { Design and Implementation of a Complex Virtual Reality System for Product } \\
\text { Design with Active Participation of End User }\end{array}$ & 2016 \\
\hline$[24]$ & $\begin{array}{l}\text { How 3D Virtual Reality Stores Can Shape Consumer Purchase Decisions: The } \\
\text { Roles of Informativeness and Playfulness }\end{array}$ & 2020 \\
\hline$[25]$ & Understanding the use of Virtual Reality in Marketing: A text mining-based review & 2019 \\
\hline$[10]$ & Virtual and augmented reality: Advancing research in consumer marketing & 2020 \\
\hline$[30]$ & Virtual Reality as a Marketing Tool & 2019 \\
\hline$[28]$ & Implementation Issues of Augmented Reality and Virtual Reality: A Survey & 2018 \\
\hline$[29]$ & $\begin{array}{l}\text { A user study trends in augmented reality and virtual reality research: A qualitative } \\
\text { study with the past three years of the ISMAR and IEEE }\end{array}$ & 2012 \\
\hline$[23]$ & $\begin{array}{l}\text { A study on the use of an immersive virtual reality store to investigate consumer } \\
\text { perceptions and purchase behavior toward non-standard fruits and vegetables }\end{array}$ & 2017 \\
\hline & & \\
\hline
\end{tabular}

We analyzed these 13 studies facing the research questions presented in section 2.2. Those two questions are addressed separately in the following subsections.

\section{Q1: How has virtual reality (VR) supporting market research in the automotive industry?}

The analyzed articles are applied in different fields of knowledge and application since the searching criteria were focused on the automotive business and VR's applicability in 
market research. Besides some papers being researched in multiple areas, three different fields of application can be identified with specific articles: a) Automotive - related to VR applicability in the automotive business, b) Retailing- relate to VR applicability on selling environments such as virtual stores, supermarket, commercial transactions or similar, and c) Advertising - the use of virtual reality for product advertising via websites. Some of these studies focused on multiple disciplines rather than just one.

Several uses of VR in the automotive industry have been discovered in the literature, including: a) technical design review [9] [19] [20] [21], where automaker employees apply VR to study design evaluations among others; b) manufacturing feasibility analysis [9], where VR is used to understand early in the development process if parts can be assemble and/or transported without issues, etc.; c) driving simulations and habits [9] [20] where automaker employees virtually simulate driving habits, driving vehicle performance, driver distractions analysis, etc.; d) training [9] [22] where training on car service, maintenance procedures, assembly process, dealers employees interaction with new vehicles, etc. and e) ergonomics [8] [20] where automaker employees understand if the vehicle is ergonomically performing in relation to end users requirements or if automakers employees can manufacturer the vehicle in an ergonomic way, preventing them from injury during their day to day work.

Since the area of research of this article is the automotive marketing research performed with end users, it is crucial to understand the type of users who were researched in the articles selected through the systematic literature review as long as they identify learnings that can apply to product development.

All studies on the retailing and advertising fields were conducted with end-users, whilst those in the automotive field of application were based on input from automaker employees. There are limited mentions of VR tools applications with end automobile customers in the various application articles. For example, virtual reality is applied to a few procedures involving customers, although it is unclear whether the authors mean that applications are conducted by the end customer or by skilled automakers representatives mimicking client behavior [9].

In the retailing application field, research has mostly concentrated on understanding consumer behavior in virtual environments and the corresponding purchase inclinations. The research was mostly conducted through the purchase of minor goods such as fruit [23] or supermarket items. A comparable application is used with automotive users during the pre-purchase process, mostly to allow customers to virtually select vehicle choices or models [10].

Because cars have a significant cost for their buyers, the study attempted to understand the various degrees of VR application based on the product cost. Marketing research applications and studies on more expensive things such as furniture [24] and even pricey 
products like as houses [25] is addressed by the literature, but not product design applications.

In the articles selected for our study, we discovered various forms of analysis. We grouped them as follows: a) VR improvements, referring to research conducted in an application or environment where virtual reality is already in use, with the purpose of improving its performance, such as switching the virtual reality application from non-immersive to immersive; b) Systematic Reviews, comprising literature surveys that incorporates knowledge from various articles, studies, and fields; c) A x B comparisons, that compares multiple virtual environments or a virtual environment with a physical one (with the virtual environment being a perception of physically being there in a non-physical setting); and d) Surveys of virtual reality users, that collects their knowledge and experience using specific questionnaires (for example, surveys done with experts from automakers on current VR applications and their anticipated prospects in a range of commercial domains).

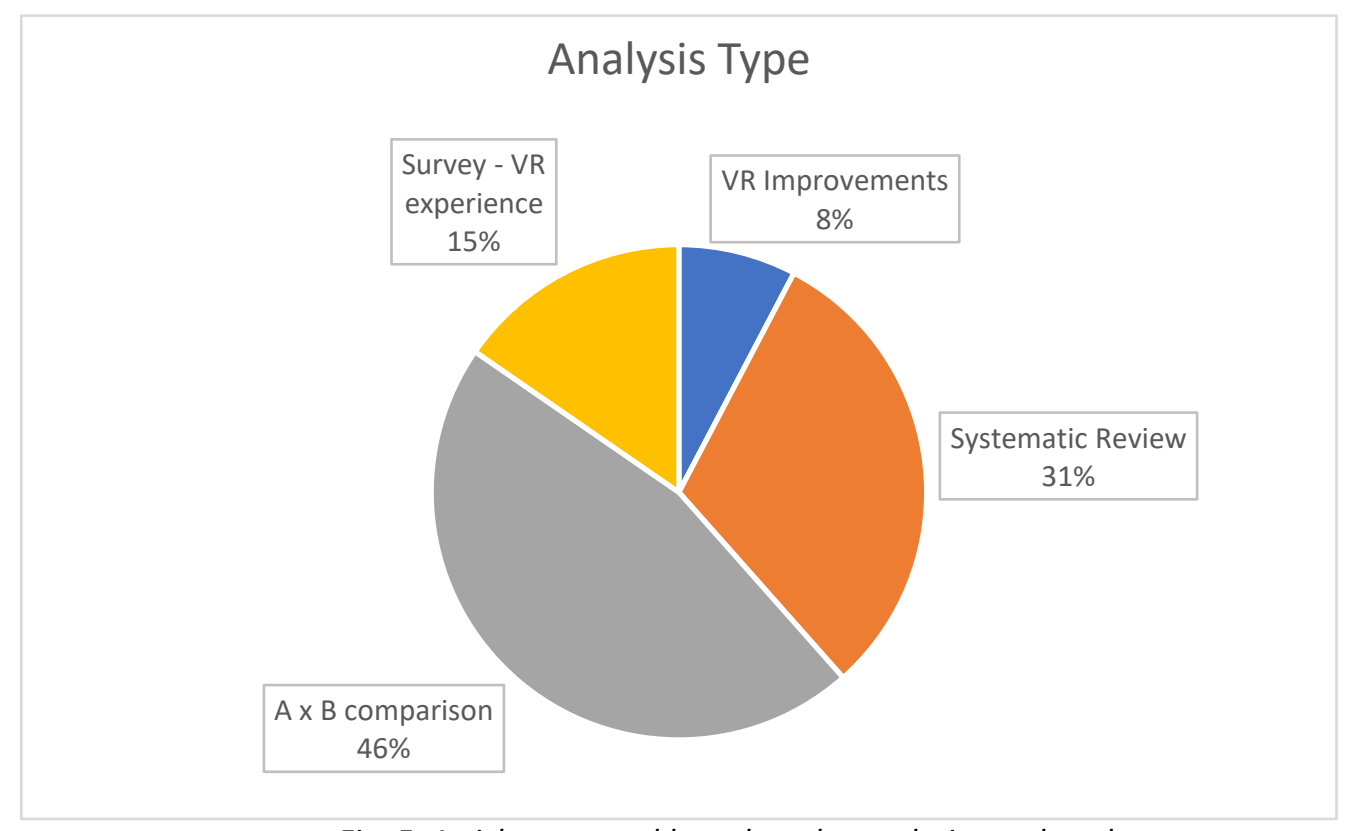

Fig. 5. Articles grouped based on the analysis employed

Table 2 illustrates the connection between the field of application and users, as well as the type of analysis. 
Table 2. Connection between the field of application and the type of analysis.

\begin{tabular}{|l|l|l|l|l|}
\hline \multirow{2}{*}{} & \multicolumn{4}{|c|}{ Field of Application } \\
\cline { 2 - 5 } & Automotive & Multiple & Retailing & Advertisement \\
\hline
\end{tabular}

\begin{tabular}{|l|l|l|l|l|c|}
\hline \multirow{3}{*}{ Users } & Experts & 6 & & & \\
\cline { 2 - 6 } & End Users & \multicolumn{3}{|c|}{1} \\
\hline \multirow{4}{*}{ Analysis Type } & VR Improvements & 1 & & 2 & \\
\cline { 2 - 6 } & Systematic Review & & 4 & & \\
\cline { 2 - 6 } & A x B comparison & 3 & & 2 & 1 \\
\cline { 2 - 6 } & Survey & 2 & & & \\
\hline
\end{tabular}

[10] observed that VR has the most potential for application in the marketing domains of product design and aesthetics, advertising, shopper marketing, multichannel retailing, social interactions, and co-creation of products and experiences, but features such as haptics and motions require additional research.

Q2: What are the most recent advancements, challenges, and opportunities in the use of virtual reality for automotive market research?

Virtual reality has been considered as a potential medium for assessing customer wishes and purchases. It is also acknowledged as approach used in market research to reduce costs, timing, and constraints such as physical goods relocation required for the research [9].

Virtual reality enables trials that would be impossible to do in physical study [26], such as research with large numbers of participants on rapidly decaying fresh products or research in multiple surroundings, such as alternative store setups.

The following sections highlight the challenges and opportunities afforded by VR in the automotive industry and marketing research. These inputs, based on a set of papers, address the Q2 question, providing a database for VR suitability in automobile marketing research. Limitations and opportunities are not limited to the automotive industry and may have an impact on market research utilization at various levels depending on the research purpose.

One of the issues identified in the literature is a lack of depth perception, which primarily affects small distances [20], and users complain about the inability of one eye to focus as well as the other. One suggestion for reducing this problem is to employ a rich environment with shadows and background texture and high-quality colors. The use of multisensory technology, such as haptic feedback, may give immersive feedback that solves the depth 
perception problem [20]. One of the most commonly highlighted concerns in the simulation done by engineers and designers was the quality of images or pictures [24]. According to [10], the field of view and the resolution of the visual display are key aspects for user immersion and are required for marketing research.

Color and texture issues were observed during design review evaluations, compromising user analysis [9]. Surface quality issues such as roughness, lack of or discontinuous surfaces that do not provide the visual feeling of a vehicle in front of the user [19] also impacted immersion in the analysis.

A lack of haptic input can also have a negative impact on the VR analysis. As long as reach perception is sought in VR environments' automobile engineering tasks, there should be no weight, torque, or force [20].

With VR, there is a problem with motion tracking. An ergonomics examination discovered a lack of hand motion monitoring, difficulties, and an uncomfortable body motion tracker suit [9]. Motion tracking and gesture recognition can be combined to enhance the VR experience [19]. Some VR equipment takes little training to use [19] [22], and body motion vests might be uncomfortable [19] [20]. In some areas, a lack of VR training may limit its implementation [27]. When immersive elements such as gesture detection and an immersive walk are used, user virtual immersion is increased [19].

VR navigation can produce a variety of immersion effects. [21] investigated the use of several solution concepts for 3D navigation with VR based on speech, gesture, and touchbased input modalities. Its research compared a spoken menu on the screen, a haptic interaction simulation of a virtual touchscreen, a head tracking system, a schematic 2D map in top views similar to Google Maps, and a touch screen. The study's findings confirm that touch screens and head tracking technologies provide the highest overall quality in these areas.

Lack of physical collision [20], sound and vibration feedback were lacking in some tests [9], causing the VR application to behave similarly to physical evaluation. Lack of sound feedback [20] was noted as a constraint in manufacturing feasibility studies, primarily connected to machinery movement or manual procedures that require operator response in addition to visual and haptic feedback. In rare circumstances, olfactory simulations are also required for assessing the air quality within the car.

Concerns like a lack of skilled individuals to install VR, the time it takes to become proficient, and a lack of VR knowledge were also recognized as VR. For some businesses, the cost of software and hardware is a barrier [27] [28].

As long as it can investigate distracting capabilities through the inquiry in the course, virtual context, such as a drivable scene to be evaluated in a realistic virtual world, gives a more substantial user immersion [9]. [24] also emphasized the impact of the backdrop context, 
where users may be presented with many repetitive cues and be more willing to overlook VR shortcomings such as graphic quality.

As a VR opportunity, the time it uses to develop a virtual prototype is minimal and can enhance the overall review process; virtual prototypes can be generated earlier than physical property, improving development schedule. The main advantages of using VR in the Malaysian automobile sector were reduced rework and improved manufacturing quality [27]. Physical prototypes are challenging to handle and move [9] [27].

One of the broadest opportunities for the usage of VR is cost savings. It also lowers the costs of delivering physical prototypes [9]. Virtual prototypes demand no storage space and are less expensive to build. Virtual assets decrease rework, save money, and boost productivity [27]. A VR network may improve virtual training and surveys by eliminating the requirement for user travel [9], hence improving application timing and costs.

The difficulties of recruiting survey respondents are also addressed in the research. Some research used a different recruitment approach between the comparisons. One example is the concern over combining face-to-face and online survey selection processes in the same study [24]. Some studies were conducted with respondents who may not have had the consumer target profile for the product under consideration, and in some circumstances, the quantity of participants may not have been statistically representative for the decisions that were required [28]. One of the three questionnaires evaluating automotive experience has a sample size of 240 interviews, while the other two have only eleven respondents. All four studies in the A x B comparison study had a sample size greater than 142 participants, however two have participants aged 18 to 22 years old, which may not represent the related population.

In marketing research, visual-spatial cues and graphic quality are critical [24]. Both challenges introduce participants to products they are already familiar with. The relevance of these challenges varies depending on the virtual research equipment. Participants in a 2D display and 3D web environment were found to be more aware of graphic quality than in a 3D virtual reality environment. [24].

According to [19], visual-spatial information is critical for customers to engage in virtual experiences, and the display of 3D VR notably boosted participants' awareness of visualspatial information and helped participants correctly associate with product accuracy.

Product manipulation improves perceived informativeness by enabling customers to process information selectively and focus on key information, hence boosting customer perception or product understanding. This engagement can be achieved by allowing control devices to mimic more realistic activities, such as physically examining items and feel their textures [24]. Immersive walking and gesture recognition increase product desirability and improve the virtual experience. 
Small-product studies show that virtual reality devices with product manipulation capabilities produce greater customer feedback association with the physical environment than platforms without manipulation. One of the challenges is the movement constraint that characterizes the virtual reality technology, as few devices include a lightweight wireless feature that allows users to freely move as they would in the real world [28].

To engage clients in the virtual environment, it is critical to allow them to interact with the products in the same way that they would interact in the real world. Mimicking physical activities in virtual reality, such as placing things in a basket or pushing a shopping cart in a virtual store, have the potential to boost customer engagement in the virtual environment.

Age may influence customer approval of the use of Virtual Reality. According to one study, groups between the ages of 15 and 45 have a more favorable response to the usage of virtual reality in field exploration than groups between the ages of 45 and 60 [30].

Cybersickness challenges was mentioned by a limited number of individuals [30] must be considered as long well as the current cybersecurity vulnerability of Virtual Reality [28]. Due to virtual reality hardware constraints (such as sensor placement), the dedicated physical setting needed in some applications might also provide a hurdle to marketing research [28].

In marketing research, the intuitiveness of VR system is also noted as something that must be addressed. If the system is not simple, it may necessitate one to two days of practice for inexperienced users [19].

According to a survey conducted in Malaysian automakers, the top five challenges to the adoption of virtual reality in the automotive sector are a lack of skilled individuals, time to become expert, a lack of information about VR, software cost, and hardware cost [27]. The prioritization of these challenges varies by region, and it has been observed that software and hardware costs can have a substantial influence on non-global automakers.

According to [25], one of the most important technologies is gaze tracking (eye-tracking), which can provide additional insights to regular surveys by evaluating dimensions such as satisfaction, brand preference, or enjoyment. [10] also emphasized the four primary categories of data to be acquired from VR systems to evaluate their effectiveness: objective, digital, neurophysiological, and self-reported data. These findings in the scientific papers are consistent with the patent analysis that revealed autonomous body movement and neural reaction recognition as a recent advancement of virtual reality with opportunities for automotive marketing research application. 


\subsection{Discussion}

Depending on the survey purpose, this knowledge about challenges and opportunities can be employed at various levels for car clinics. Since the clinic's goal is to determine style acceptability for customers, the most critical features in car clinics concern to the stimuli to be visually as realistic as possible with a production vehicle.

This study's articles revealed several challenges and opportunities that can be implemented to an immersive car clinic. Based on our experience with car clinics, the following items are relevant: 1) cost, 2) proximity to customers, 3) flexibility in interactions, and 4) model transportation's avoidance and challenges as 5) lack of depth perception, 6) lack of haptic perception, 7) motion perception, 8) lack of physical collision/ movement perception, 9) color and texture definition, 10) sound feedback, 11) lack of product interaction/manipulation, 12) visual-spatial, 13) graphic quality, 14) intuitiveness, 15) cybersecurity, and 16) cybersickness.

Visual-spatial and graphic quality are the most important challenges in an automobile clinic. External design and vehicle size are important factors in consumer decisions in the automotive business, and visual-spatial and graphic quality are strongly associated. The visual-spatial and graphic quality must be addressed for effective virtual reality application in a car clinic.

Graphic Quality might be sensitive to this sort of assessment since automotive marketing research focuses on vehicle design, and a sufficient final quality stimulus must be employed in car clinics so customers can provide relevant input. Customers prefer to focus on the flaws of the stimuli rather than the stimuli themselves, which may compromise the clinic's goals. When stimuli lack the quality and craftsmanship required to deliver the same final appearance as a production car, this issue is also noticed in the physical clinic. A strategy might be to select the suitable VR devices, as well as relevant skills on the crew that develops the virtual stimuli. A pre-event technical team evaluation, which is also undertaken to support physical research, can uncover these difficulties and fix them within an appropriate time frame.

The graphic quality, as well as the associated VR hardware and software, must be defined so that consumers do not lose fluidity. If this challenge is not addressed, respondents may have the feeling that they are watching a 1930s movie, with visual motions that are not at the pace required by the brain to give the user the idea that they are in a real world.

Cybersecurity, product manipulation/interaction, depth perception, and color and texture all have the potential to cause significant effect on immersive car clinic applications. Due to product confidentiality, car clinics in the automotive industry are held in a secure setting; the virtual clinic likewise demands limiting access. Cybersecurity must be carefully considered. Now there is software that can minimize the threat of illegal access during survey. 
In terms of manipulation and interaction in a virtual car clinic, customers should be able to roam around the item on their own, achieving the higher perceived amount of information required between product and customer. This would reduce any adverse effects on virtual product manipulation and engagement. A hybrid reality, which can give some level of physical manipulation and interactions connected with a virtual process, could also be a remedy to this challenge.

Environments that allow customers to wander around the virtual asset which simulates the same experience as a conventional car clinic with a physical asset should strengthen the association. To decide how realistic this experience should be, a balancing between the needs of a larger VR area and the advantage of customers being more immersed in the clinic should be undertaken.

Many simulation opportunities, such as opening a door or manipulating mirrors, may be accomplished virtually, but do necessitate refinement in Virtual Reality stimuli and potentially more robust equipment to process such data without losing customers' feeling of movement. Another advantage of virtual reality is the opportunity to conduct the clinic in a considerably more appealing atmosphere than is afforded by physical survey. VR can provide the buyer the impression that they are in a friendlier location, such as a park, vast showroom, open atmosphere, and so on. This approach is challenging and costly to implement in a physical car clinic, especially when confidentiality and security considerations are addressed.

Customers prefer not to come too close to the car when depth perception concerns are most apparent, therefore that may not be an issue in market research for exterior design testing experimentation. Customers may be bothered by vehicle interior feedback because most customer interfaces and verifications are performed at close proximity to the stimuli. The specification of the Head Mounted Display (HMD) may also lessen depth perception concerns, so it is critical to determine the best hardware depending on the intended survey outcome.

Different textures and colors are presented with small samples or 2D images in physical stimuli, rather than in a vehicle environment, because this would considerably increase the complexity of stimuli to be done. Virtual reality enhances the opportunity to test various colors and textures in the vehicle surroundings. Through sensors, current VR technology may provide visual texture and basic haptic experience. This difficulty may be readily overcome by combining virtual and actual surroundings. Small physical samples of color and texture taken outside of the virtual environment can be used in the same manner as physical clinics are now operated without the need for several pricey physical stimuli. This can be utilized if VR gear is not available or to lower the expense of virtual vehicle clinics.

Some difficulties should have a significant impact on car clinics. Customers, for example, must be immersed in a virtual setting comparable to what they would be in the real world 
for vehicle clinics. On that basis, the intuitiveness of the VR equipment, cybersickness, haptic and physical collisions should all be considered.

Concerns about intuitiveness in immersive car clinics can be alleviated by selecting appropriate VR devices and delivering consumer instruction prior to the survey. Respondents can experience cybersickness, which is most seen after lengthy periods of use. Car clinics are normally accomplished in a couple hours with some interruptions, such as coffee breaks. These pauses can be timed in a way that minimizes cybersickness; similarly, paying attention to participants' well-being allows for schedule changes.

Customers' immersion in the event would be enhanced via haptic feedback and physical collision. Most interactions with prototypes at car clinics are directed by hand-eye coordination, allowing a customer's eyesight to complement hand movement and touch; haptic sensor gloves might support with this perception in a virtual environment. Another option is to utilize a hybrid technology, also known as mixed reality, in some parts of the automotive industry, which combines virtual reality with a simple physical asset that customers can touch.

The challenges associated with motion perception, sound feedback, and the physical environment necessitated by VR hardware should be of little concern in car clinics. Clinics are held in workshops that require space for four to eight vehicles, which is far greater than what VR hardware requires. Because physical prototypes seldom give sound feedback, it is usual in traditional car clinics to instruct participants not to evaluate particular stimulus performance, such as door shutting sound or similar, due to manufacturing and material limitations. However, if sound is a characteristic to be assessed, having hand gloves with multi-sensor and audio connections might improve the survey.

The primary advantages of employing virtual reality in car clinics are the lowering of development expenses and the reduction of time. Several physical prototypes, such as ergonomic prototypes, have been shown in studies to be replaced by virtual prototypes with cost savings and improved time analyses. VR also allows for the investigation of vehicle differentiation (variety, alternatives, and content) with a single stimulus and clinic encounter, allowing for greater flexibility in interactions.

Customers are also prevented from harming physical prototypes during car clinics, which is a recurring occurrence that jeopardizes the survey. Virtual reality also eliminates the need to transport confidential physical stimuli from the fabrication shop to a showroom. The avoidance of these difficulties lowers the expenses of the car clinic. These opportunities are connected to model transportation avoidance and customer proximity.

Aside from the employment of virtual reality in the automotive industry, we found few applications of end automotive customers in the early product development phase in the literature. This might be explained by the key challenges mentioned before, such as a shortage of skilled workers in the market and high hardware and software costs. This 
scenario may change because of the growth of VR-capable professionals in recent years, as well as the decreasing costs of software and hardware.

Another possible explanation for the scarce literature on virtual reality applications in car clinics is the automotive industry's secrecy around product development and marketing research. Automakers may be exploring and possibly implementing virtual reality alternatives, but data is not yet publicly available. The paucity of virtual market research studies with products with similar price to vehicles may not give the confidence to implement this technology in car clinics.

The automotive business has a variety of challenges and possibilities. There is no one-sizefits-all approach for implementing Virtual Reality methodology. The findings show that, depending on the marketing research goals, the challenges highlighted in a wider variety than the opportunities may be prevented or managed by simple measures.

For most car clinics, a combination of virtual reality and low-cost physical prototypes tends to be the most cost-effective approach. Following that, a set of challenges and opportunities, as well as strategic suggestions, on how to employ virtual reality technology for automotive applications, which are likely to be applicable in other domains.

\section{Conclusion}

The automobile sector has been under pressure to reduce time to market and increase product definition accuracy. Virtual reality is a powerful tool to engage with customers from the early stages of product development through after-sales support. The application of Virtual Reality in car clinics might be a cost saving measure and shorten cycle time in the automotive industry.

Technology research greatly contribute to identifying opportunities and challenges in employing virtual reality in car clinics through scientific and technological knowledge.

According to the patents that were examined, there is no obvious trend on virtual reality applications to marketing research. In terms of the origin of active patented technology, there is a concentration in developed countries, with the United States and Japan dominating. A few inventors' organizations monopolize active patents, with five groups accounting for 49 percent of total papers, four of which are from the United States. We observed that patents could be classified into four sorts of applications: automatic feedback, customer profile, advertisement, and virtual stores, with automatic feedback being the most prominent throughout the years and potentially valuable in virtual car clinics.

The following sixteen attributes resulted from a review of the literature on the opportunities and challenges of using VR for automotive market research. As opportunities, 1) cost, 2) proximity to customers, 3) flexibility in interactions, and 4) model transportation's avoidance and challenges as 5) lack of depth perception, 6) lack of haptic perception, 7) 
motion perception, 8) physical collision/ movement perception, 9) color and texture definition, 10) sound feedback, 11) product interaction/manipulation, 12) visual-spatial, 13) graphic quality, 14) intuitiveness, 15) cybersecurity, 16) cybersickness.

As a result, we found that virtual reality in car clinics might be employed, but factors such as hardware and software specification, stimulus quality, research aims, and Hybrid Reality suitability, among others, must be properly accounted.

We suggest further research to better understand the correlation of virtual reality in car clinics research compared to physical research.

\section{References}

[1] Jerald, J. (2015). The VR book: Human-centered design for virtual reality. Morgan \& Claypool.

[2] Hamilton, D., McKechnie, J., Edgerton, E., \& Wilson, C. (2021). Immersive virtual reality as a pedagogical tool in education: a systematic literature review of quantitative learning outcomes and experimental design. Journal of Computers in Education, 8(1), 1-32.

[3] Genaro, L. E., \& de Oliveira Capote, T. S. (2021). Use of Virtual Reality in Dentistry: Literature Review. Odovtos-International Journal of Dental Sciences, 23(2), 33-38.

[4] Cao, C., \& Cerfolio, R. J. (2019). Virtual or Augmented Reality to Enhance Surgical Education Planning and Surgical. Education and the Thoracic Surgeon, An Issue of Thoracic Surgery Clinics, Ebook, 29(3), 329-337.

[5] Sidani, A., Dinis, F. M., Sanhudo, L., Duarte, J., Baptista, J. S., Martins, J. P., \& Soeiro, A. (2021). Recent tools and techniques of BIM-based virtual reality: A systematic review. Archives of Computational Methods in Engineering, 28(2), 449-462.

[6] Fortune Business Insight. (2019). Virtual Reality In Automotive Market Size, Share \& Industry Analysis, By Component (Hardware, Software, Content), By Application (Designing and Prototyping, Training, Virtual Reality Showrooms, and Research and Development), and Regional Forecast, 20192026. Retrieved July 17, 2020, from https://www.fortunebusinessinsights.com/industry-reports/virtualreality-vr-in-automotive-market-101702

[7] Wolfartsberger, J. (2019). Analyzing the potential of Virtual Reality for engineering design review. Automation in Construction, 104, 27-37.

[8] Hashim, A. M., Whitfield, T. W., Jackson, S., \& Effendi, R. A. A. R. (2012). The application of experimental aesthetics to a marketing research method in the automotive industry.

[9] Lawson, G., Salanitri, D., \& Waterfield, B. (2016). Future directions for the development of virtual reality within an automotive manufacturer. Applied ergonomics, 53, 323-330.

[10] Wedel, M., Bigné, E., \& Zhang, J. (2020). Virtual and augmented reality: Advancing research in consumer marketing. International Journal of Research in Marketing, 37(3), 443-465.

[10] Henriques, A. C., \& Winkler, I. (2020, November). Automotive Marketing Research and Virtual Reality: A Systematic Literature Review. In 2020 22nd Symposium on Virtual and Augmented Reality (SVR) (pp. 339-345). IEEE. 
[11] Blackman, M. (1995). Provision of patent information: a national patent office perspective. World Patent Information, 17(2), 115-123.

[12] Lee, C., Kang, B., \& Shin, J. (2015). Novelty-focused patent mapping for technology opportunity analysis. Technological Forecasting and Social Change, 90, 355-365.

[13] Maricato, J. D. M., \& Noronha, D. P. (2012). Indicadores bibliométricos e cientométricos em CT\&I: apontamentos históricos, metodológicos e tendências de aplicação. J. HAYASHI, MCPI; LETA (Ed.), Bibliometria e cientometria: reflexões teóricas e interfaces, 21-41.

[14] Santos-Neto, J. F., Oliveira, F. O., Hodel, K. V., Fonseca, L., Badaró, R., \& Machado, B. A. (2021). Technological advancements in monoclonal antibodies. The Scientific World Journal, 2021.

[15] Speziali, M. G., \& Sinisterra, R. D. (2015). TECHNOLOGICAL INFORMATION SEARCHING BASED ON PATENT DATABANK: CASE STUDY OF IONIC LIQUIDS IN BRAZIL. Química Nova, 38(8), 1132-1138.

[16] Pradeep, A., Knight, R. T., \& Gurumoorthy, R. (2018). U.S. Patent No. 9,886,981. Washington, DC: U.S. Patent and Trademark Office.

[17] Register, T. L., Trottie-register, D., \& Trottie, C. (2018). U.S. Patent No. 9,881,310. Washington, DC: U.S. Patent and Trademark Office.

[18] Edwards, M. (2012). U.S. Patent No. 8,341,022. Washington, DC: U.S. Patent and Trademark Office.

[19] Górski, F., Buń, P., Wichniarek, R., Zawadzki, P., \& Hamrol, A. (2016). Design and implementation of a complex virtual reality system for product design with active participation of end user. In Advances in Human Factors, Software, and Systems Engineering (pp. 31-43). Springer, Cham.

[20] Lawson, G., Salanitri, D., \& Waterfield, B. (2015, August). Vr processes in the automotive industry. In International Conference on Human-Computer Interaction (pp. 208-217). Springer, Cham.

[21] de Clerk, M., Schmierer, G., Dangelmaier, M., \& Spath, D. (2017, December). Interaction techniques for virtual reality based automotive design reviews. In International Conference on Virtual Reality and Augmented Reality (pp. 39-48). Springer, Cham.

[22] Borsci, S., Lawson, G., Burgess, M., \& Jha, B. (2015, August). Early prototype assessment of a new virtual system for training procedural skills of automotive service operators: LARTE tool. In International Conference on Human-Computer Interaction (pp. 135-143). Springer, Cham.

[23] Verhulst, A., Normand, J. M., Lombart, C., \& Moreau, G. (2017, March). A study on the use of an immersive virtual reality store to investigate consumer perceptions and purchase behavior toward non-standard fruits and vegetables. In 2017 IEEE Virtual Reality (VR) (pp. 55-63). IEEE.

[24] Kang, H. J., Shin, J. H., \& Ponto, K. (2020). How 3D virtual reality stores can shape consumer purchase decisions: the roles of informativeness and playfulness. Journal of Interactive Marketing, 49, 70-85.

[25] Loureiro, S. M. C., Guerreiro, J., Eloy, S., Langaro, D., \& Panchapakesan, P. (2019). Understanding the use of Virtual Reality in Marketing: A text mining-based review. Journal of Business Research, 100, 514-530. 
[26] Lombart, C., Millan, E., Normand, J. M., Verhulst, A., Labbé-Pinlon, B., \& Moreau, G. (2020). Effects of physical, non-immersive virtual, and immersive virtual store environments on consumers' perceptions and purchase behavior. Computers in Human Behavior, 110, 106374.

[27] Mousavi, M., Aziz, F. A., \& Ismail, N. (2012, March). Opportunities and constraints of virtual reality application in international and domestic car companies of Malaysia. In 2012 UKSim 14th International Conference on Computer Modelling and Simulation (pp. 273-277). IEEE.

[28] Kumari, S., \& Polke, N. (2018, August). Implementation Issues of Augmented Reality and Virtual Reality: A Survey. In International Conference on Intelligent Data Communication Technologies and Internet of Things (pp. 853-861). Springer, Cham.

[29] Kim, S. J. J. (2012, August). A user study trends in augmented reality and virtual reality research: A qualitative study with the past three years of the ISMAR and IEEE VR conference papers. In 2012 International Symposium on Ubiquitous Virtual Reality (pp. 1-5). IEEE.

[30] Singh, H., Singh, C., \& Majumdar, R. (2019). Virtual reality as a marketing tool. In Emerging Trends in Expert Applications and Security (pp. 445-450). Springer, Singapore. 